\title{
Continuous suture hepaticojejunostomy is economical with similar long term results as interrupted suture technique: An audit of a prospective database of 556 hepaticojejunostomies
}

\author{
Rajan SAXENA*, Nalini Kanta GHOSH, Saurabh GALODHA
}

Department of Surgical Gastroenterology and Center for Hepatobiliary Diseases and Transplantation, Sanjay Gandhi Postgraduate Institute of Medical Sciences, Lucknow, India

Introduction: Hepaticojejunostomy $(\mathrm{HJ})$, a standard method of bilioenteric anastomosis, is done with interrupted sutures by most surgeons. This audit of a prospective database compares the safety, economics, short term and long term outcome of continuous suture $\mathrm{HJ}$ (CSHJ) and interrupted suture HJ (ISHJ).

Methods: An audit of a prospecrive database of all HJ performed between January 2014 and December 2018 after IEB approval. Patients with type IV or higher biliary strictures, duct diameter $<8 \mathrm{~mm}$ and/or associated vascular injury and liver transplant recipients were excluded. Patient demographics, diagnosis, pre-operative parameters, intra-operative findings, type and number of sutures, suturing time, and postoperative morbidity (Clavien Dindo) were recorded, and patients followed up to 60 months. McDonald's grade A and B were considered as good outcome. Cost of suture (polydioxanone) 3-0/5-0 mean cost - ₹686/length, polyglactin 3-0, 4-0 mean cost - ₹486/length), operating room time (₹5,000/hour) were considered for comparison of economics of both techniques. Statistical analysis done on SPSS 22 software.

Results: 556 eligible patients - 468 with ISHJ and 88 with CSHJ analyzed. 258 (54\%) had benign and 300 (46\%) had malignant pathology. The groups were similar. PDS sutures dominated in CSHJ. Number of sutures, cost, time, and postoperative bile leak was significantly more in ISHJ group. Fifty four patients had bile leak (6 CSHJ and 48 ISHJ). There were 16 mortalities (3 CSHJ, 13 ISHJ) due to septic shock. Morbidity was comparable according to Clavien Dindo grading. Anastomotic stricture rate was comparable.

Conclusions: $\mathrm{CSHJ}$ is safe, economic and worthy of routine practice. 